\title{
Information visualization analysis based on historical data
}

\author{
Yukun Zhang ${ }^{1} \cdot$ Bei Wu ${ }^{1} \cdot$ Lifeng $\operatorname{Tan}^{1} \cdot$ Jiayi Liu ${ }^{2}$
}

Received: 24 December 2020 / Revised: 23 April 2021 / Accepted: 5 May 2021 /

Published online: 17 May 2021

(C) The Author(s) 2021

\begin{abstract}
Visual expression is increasingly used in historical research due to its intuitiveness and distinctness. However, most of the common research contents focus on the spatial concept, but lack the visualization analysis of the attribute characteristics of the research elements. In order to achieve this goal, based on a case study of the coastal military defense system in Ming Dynasty, the Geographic Information System (GIS) platform was adopted to reconstruct the historical map and its spatial data were extracted. On this foundation, the attribute characteristics of the military settlements, accessibility, was quantified by constructing a hierarchy evaluation model, and then the results were projected into the spatial geographic coordinates to realize the visualization of the accessibility of the military settlements in Ming Dynasty. The results showed that the combined method of quantification and visualization not only enabled more comprehensive and intuitive display of historical information, but also promoted data extraction and correlation analysis, creating a possibly for more in-depth future research.
\end{abstract}

Keywords Historical data · Visualization · Data quantification · GIS · Coastal defense system

\section{Introduction}

For hundreds of years, people have been using characters to record and convey history. However, characters have their inherent limitations, which restrict the way people think and communicate. The rise of the computer has fundamentally changed how human beings receive

Yukun Zhang and Bei Wu contributed equally to this work.

Lifeng Tan

tanlf_arch@163.com

Jiayi Liu

liujiayi2012@126.com

1 Key Laboratory of Information Technology for Architectural Heritage Inheritance of the Ministry of Culture and Tourism of China, School of Architecture, Tianjin University, Tianjin, China

2 School of Marine Science and Technology, Tianjin University, Tianjin, China 
and process information. Digital environments and virtual reality are adding a third dimension to communication and creating a new visual language [17]. In particular, the development of historical geography in the twentieth century, under the intervention of GIS and spatial analysis techniques, provided historians with a new method that regarded visualization as important as writing, and continued to diversify. History can also be visually reconstructed for more effective and accurate expression.

A large number of historical geography researches relying on visualization methods emerged, such as the most common historical map reconstruction $[8,12,15,19,24]$, historical data statistics [5, 6, 18, 25], and historical literature narrative analysis [1, 13, 14, 23]. This kind of research on a certain element in a certain area within a certain period of time is the usual way of conventional historical visualization research. This way has been continued in the past few decades, forming a simple and convenient routine research. However, from the perspective of contemporary historical geography, things cannot be studied as a special or isolated phenomenon; instead, their general characteristics shall be investigated, especially the complex interrelationships between various components [9]. So, on the basis of historical research on individual elements of human geography, a further step is taken, meaning that regional and comprehensive regional historical geography is considered.

The research on comprehensive historical geography elements should not be limited to the spatial concept; instead, the location of each element shall be restored, and their relevance, attribute characteristics and formation causes shall also be explored to find out the rules. However, these attribute characteristics are so abstract that they are often understood through systematic analysis of professionals. Conventional visualization methods alone generally do not work. Therefore, it is necessary to use quantitative analysis to translate attribute characteristics into numerical values, and then realize their visualization. But most of the geographical phenomena in history cannot be restored through field investigations, which only rely on documentary records. Pitifully, existing records often provide incomplete descriptions. Hence, it is quite difficult to carry out quantitative analysis and draw precise conclusions.

To solve this problem, this article conducts a case study of a complex system, namely, the coastal military defense system in Ming Dynasty, and tires to visualize its attribute characteristic "accessibility". First of all, the spatial factors affecting the accessibility of military settlements are explored, and an evaluation model is established for quantitative analysis of the accessibility. Next, names and relative positions of the military settlements recorded in local chronicles and atlas are utilized to distinguish the direction of each facility, and the spatial concepts are visualized to rebuild the distribution map of the coastal military defense system in Ming Dynasty. Thus, their spatial relationships can be extracted by relying on GIS analysis tools for data extraction. Then, the spatial data is substituted into the hierarchy evaluation model to get the quantitative data which can be used to measure the accessibility of military settlements. Finally, the quantification results are mapped into space, and a visualized outcome of the accessibility of military settlements is obtained, making it possible for us to determine its formative causes and rules.

\section{Study materials}

\subsection{Study object}

After the founding of the Ming Dynasty, the maritime situation was not calm as it seemed to be. The remnant rebel forces and Japanese pirates constantly invaded the southeastern coastal 
areas, and even colluded with each other, which brought great trouble to the Ming Dynasty. In order to eradicate rampant Japanese pirates, maintain maritime prohibition policies and protect the waterways, the Ming Dynasty began to build the military settlements to a large scale, and finally formed a systematic coastal military defense system for the first time in Chinese history, which constituted two defense lines in the south and north of the Ming Dynasty together with the Great Wall defense system [4].

Structured in levels, strict in grades and varied in scale, the coastal military defense system of the Ming Dynasty shaped an orderly spatial layout on the coastline ranging from the east of the Liaoning Province to Guangdong and Hainan, serving as a typical complex system in the historical period. The Wei cities and Suo cities (Wei-Suo, 卫所) formed the main defense framework; the patrol and inspection agencies (巡检司) played the role of investigation; the post stations (驿站) and urgent delivery stations (急递铺) acted as the transit stations during wartime; the beacon towers (烽埃) constituted the meticulous enemy situation forecast system. They were not only independent but also collaborative, taking charge of protecting the territory of the Ming Dynasty and the safety of the people.

The concept "accessibility" was first proposed in the field of transportation by Walter G. Hansen in 1959, referring to the interaction opportunity of nodes in transportation network [7]. Informally, it can be understood as the ease of heading from one point to another. Accessibility is an important part of spatial analysis. In recent years, many archaeologists at home and abroad have applied accessibility analysis to settlement research [10, 11, 21], and provided scientific methods for spatial analysis with computer-aided means.

For the coastal military defense system of the Ming Dynasty, the settlements were closely related and mutually dependent, highlighting the significance of the accessibility of the settlements. It mattered a lot to the delivery speed of warning signals, military information and materials, guaranteeing victories in the war, largely deciding the defensive strength of the coastal military defense system. While exploring the accessibility of military settlements in the Ming Dynasty, these key factors shall be taken into account: the beacon towers for observing the enemy's situation and alarm transmission, the post stations and urgent delivery stations for transmitting official documents and military materials, and the main defense units, Wei cities and Suo cities.

In general, by visualizing the accessibility characteristics of military settlements, the layout emphases of the coastal military defense system in Ming Dynasty, as well as the relationship between the settlements at all layers, were unveiled to the world.

\subsection{Study area}

This paper takes Ningbo (宁波) Prefecture, a coastal area in the Ming Dynasty, as the research area. Ningbo Prefecture was located in the east of Zhejiang (浙江) Province, bordering the sea in the east, south and north, with many islands along the coast. In the Ming Dynasty, there were five counties in Ningbo, namely Yin (鄞) County, Cixi (慈溪) County, Fenghua (奉化) County, Dinghai (定海) County and Xiangshan (象山) County, as well as a prefectural city situated in Yin County, which was the highest administrative unit of Ningbo (Fig. 1) [19]. From the military point of view, Ningbo had always been a gateway for Japanese pirates to invade the mainland from the sea. Once Ningbo fell, the military situation in the whole southeastern coastal area would be seriously affected. Therefore, the Ming government attached great importance to the construction of military defense system in Ningbo, which is a typical case of coastal military defense research. Hence, comprehensive local chronicles and military monographs of Ningbo also lays a foundation for this visualization study. 


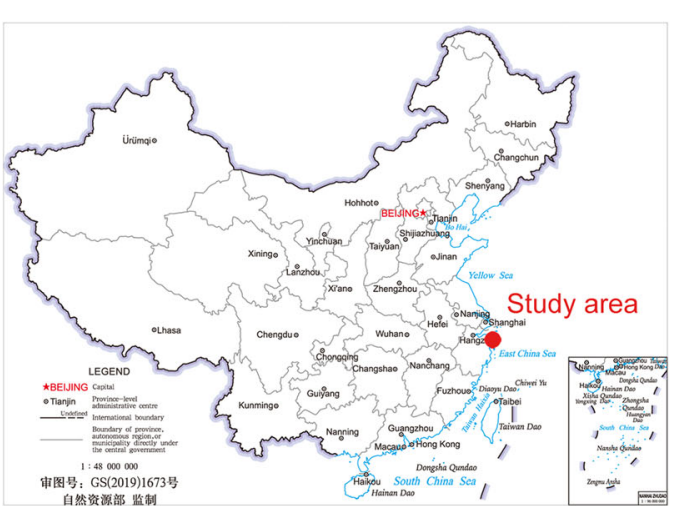

(a)

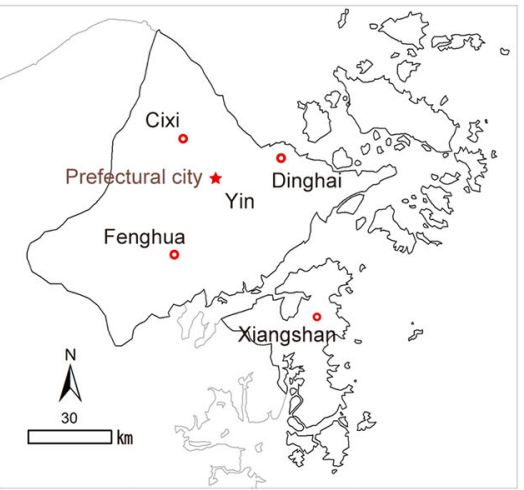

(b)

Fig. 1 Location (a) and administrative divisions (b) of the study area. (a) is provided by Ministry of Natural Resources of China (Approval number: GS(2019)1673)

\subsection{Data sources}

All the data of the military defense system used in this study are taken from Chou Hai Tu Bian (筹海图编) [30] and Ningbo Annals of Jiajing Period (嘉靖宁波府志) [28]. The data of Wei cities, Suo cities and beacon towers are collected from Chou Hai Tu Bian, which is a coastal military atlas compiled by Ruozhen Zheng (郑若曾) during Jiajing (嘉靖) period of Ming Dynasty. The atlas details the number and names of the Wei cities, Suo cities and beacon towers in the coastal areas, which can be rated the most comprehensive historical record for the coastal military facilities in the Ming Dynasty. The data of post stations and urgent delivery stations come from Ningbo Annals of Jiajing Period compiled by Shiche Zhang (张时徹) during Jiajing period of Ming Dynasty. The annals records the geography, history, transportation, military and other aspects of each region, including the names, routes and probable locations of post stations and urgent delivery stations, providing lots of useful information for the descendents to know about the post system of Ningbo. All the original data are presented in the form of written words.

According to the data extracted from Chou Hai Tu Bian and Ningbo Annals of Jiajing Period, there were about 3 Wei cities, 8 Suo cities, 91 beacon towers, 3 post stations and 85 urgent delivery stations in Ningbo of Ming Dynasty (Tables 1 and 2).

In addition, the $30 \mathrm{~m}$ resolution Digital Elevation Model (DEM) map of Ningbo is also used in this study, which is provided by Geospatial Data Cloud site, Computer Network Information Center, Chinese Academy of Sciences (http://www.gscloud.cn).

\section{Study methods}

\subsection{Hierarchy evaluation model establishment}

The accessibility of military settlements in Ming Dynasty can be understood as the convenience degree from one settlement to the nearest one. Then, which contents need to be transferred from one settlement to another? The most common ones are alarm signals, materials and information, etc. According to the contents that are delivered, in order to judge 
Table 1 Wei cities, Suo cities and beacon towers statistics

Wei cities \& Suo Bencon towers with the jurisdiction of Wei cities and Suo cities cities

Dasong Suo Guanhai Wei Longshan Suo Dinghai Wei

Chuanshan Suo Guoju Suo Zhoushan Suo

Changguo Wei Qiancang Suo Juexi Suo Shipu Suo
Daqian, Kunting, Hengshan, Huangyan, Ci’ao, Ha'ao, Jianqi, Gangkou Xiangtou, Guashi, Xilongshan, Xinpu, Guyao, Xilongwei

Shigongshan, Longtou, Longwei, Shitang, Qingxi

Gaoshan, Zhushan, Xiaoshan, Luci, Zhaobaoshan, Dagushan, Zhangshipu, Dajian'gang, Dayuwan, Changshanfeng, Jiyu, Wangjialu

Qitou, Suohou, Baifeng, Xishan

Sheng'ao, Gaoshan, Meishan, Guanshan, Xiala

Waihu, Shiqiang, Baojia, Shitong, Lujing, Pusha, Xishan, Dingchi, Chishi, Jiedai, Qi'ao, Xiaozhan, Diaoyu, Chengjia, Shichan, Xiepu, Zhoushan, Shenjiamen, Langjiaqi, Yuanjiaqi, Luotou, Ganlan, Sanjiang

Huangsha, Song'ao, Renyi, Hejialan, Houshan, Chutou, Wushi, Qianshan, Chikan Qinglei, Pumenling, Dongmen, Zhongbao, Qianshan, Tuci, Shanmuyang Gongyu, Banling, Zhao'ao, Shaling, Pingfeng, Yuquan, Wailing Dajinshan, Tuwan, Song'ao, Xia'ao, Majiashan

whether the transmission of alarm signals, materials, and information between two settlements is efficient and unobstructed, it is necessary to measure the accessibility of different transmission modes and routes. Problems should be stratified and methodized based on the characteristics of different transmission modes, and the influencing factors should be classified and listed hierarchically, so as to make a rational evaluation of the importance of each indicator.

Firstly, the first-level indicators are sorted out. According to the contents that need to be transmitted, the influencing factors of accessibility between settlements can be confirmed. The alarm signals were transmitted by beacon towers; the materials were transported by post stations; the information was delivered by urgent delivery stations or by soldiers in the Wei cities and Suo cities. Therefore, the first-level indicators can be divided into 3 groups, namely, the accessibility between beacon towers and Wei-Suo, the accessibility between post stations and Wei-Suo, and the accessibility among Wei-Suo, respectively.

Table 2 Post stations and urgent delivery stations statistics

\begin{tabular}{|c|c|c|}
\hline Counties & $\begin{array}{l}\text { Post stations } \\
\text { (Yi) }\end{array}$ & Urgent Delivery Stations $(\mathrm{Pu})$ \\
\hline Yin & Siming Yi & $\begin{array}{l}\text { Fuqian Pu, Chengdong Pu, Fuming Pu, Shengdian Pu, Huiqian Pu, Daji Pu, Yuwang } \\
\mathrm{Pu} \text {, Sanxi Pu, Huanglong Pu, Dengjia Pu, Dasong Pu, Miaodun Pu, Huoba Pu, } \\
\text { Guling Pu, Dongqiao Pu, Xinqiao Pu, Yanqiao Pu, Xintang Pu, Jiatang Pu, } \\
\text { Jing'an } \mathrm{Pu} \text {, Xin Pu, Zhuanqiao Pu }\end{array}$ \\
\hline Cixi & Chejiu Yi & $\begin{array}{l}\text { Xiqian Pu, Jiatian Pu, Xidu Pu, Tongqiao Pu, Niqiao Pu, Jiashan Pu, Luojia Pu, } \\
\text { Jiangjia Pu, Taiping Pu, Songpu Pu, Guanhai Pu }\end{array}$ \\
\hline Fenghua & Lianshan Yi & $\begin{array}{l}\text { Xianqian Pu, Longtan Pu, Shangtian Pu, Shuangxi Pu, Fangmen } \mathrm{Pu} \text {, Shanhuang Pu, } \\
\text { Tanxu Pu, Jinzhong Pu, Nandu Pu, Chenqiao Pu, Changpu Pu }\end{array}$ \\
\hline Dinghai & / & $\begin{array}{l}\text { Xianqian Pu, Jiagang Pu, Kongshu Pu, Xin'ao Pu, Changshan Pu, Chenhua Pu, } \\
\text { Chuanshan Pu, Zhuling Pu, Ci'ao Pu, Menyan Pu, Guoju Pu, Dongla Pu, Xiala } \\
\text { Pu, Kunting Pu, Qingshui Pu, Yongfu Pu, Kongpu Pu, Guantuan Pu, Qutang Pu, } \\
\text { Xujia Pu, Suoqian Pu, Longtou Pu, Zhoushan Pu }\end{array}$ \\
\hline Xiangshan & / & $\begin{array}{l}\text { Xianqian Pu, Huoshao Pu, Sanjiao Pu, Hutou Pu, Shanmuyang Pu, Qiancang Pu, } \\
\text { Dajing Pu, Laoling Pu, Yingjia Pu, Dongxi Pu, Ha'ao Pu, Jiaowan Pu, Jiming Pu, } \\
\text { Weiqian Pu, Yu'nv Pu, Juexi Pu, Baiyan Pu, Fangqian Pu }\end{array}$ \\
\hline
\end{tabular}


Secondly, further classification is made based on the characteristics of each transmission mode. Since beacon towers relied on fire and smoke to report the enemy's situation, beacon towers must be situated for a coherent information flow within the effective reachable range of vision or hearing, so as to transmit enemy situation effectively. Therefore, three indicators affected the accessibility between beacon towers and Wei-Suo, namely, the number, average observation distance and vision field of beacon towers within the jurisdiction of Wei-Suo respectively. Post stations were equipped with horses to transmit, so the only indicator of judging the accessibility between post stations and Wei-Suo is the distance. While the transmission among Wei-Suo hinged on two ways: urgent delivery stations relied on the couriers to deliver information by walking; soldiers in the Wei cities and Suo cities transmitted information on foot or on horseback. Hence, the distance of two routes needs to be measured in the accessibility among Wei-Suo.

According to above-mentioned analysis, a hierarchy evaluation system is established for the accessibility of military settlements in the Ming Dynasty (Table 3).

Finally, the importance of each indicator in the hierarchy evaluation system need to be estimated. In order to ensure the accuracy of the results, several experts in the study of military defense system are invited to judge the importance of each indicator. The result is: the importance of different indicators in each classification group is equal. That is, B1, B2, B3 contribute equally to $\mathrm{A} ; \mathrm{C} 1, \mathrm{C} 2, \mathrm{C} 3$ contribute equally to $\mathrm{B} 1 ; \mathrm{C} 5, \mathrm{C} 6$ contribute equally to $\mathrm{B} 3$. Then, the weight of each indicator is obtained, establishing the hierarchy evaluation model of the accessibility of coastal military settlements in the Ming Dynasty (Fig. 2). In this study, the hierarchy evaluation model is used to quantify the accessibility characteristics of different settlements of Ningbo in Ming Dynasty.

\subsection{Spatial analysis and data quantification based on GIS}

\subsubsection{Historical map reconstruction}

The written historical data can not be directly used to calculate the accessibility of military settlements of Ningbo in Ming Dynasty, so it is necessary to extract the spacial data of each indicator with the help of GIS tools. Then, the military facilities in Tables 1 and 2 need to be localized and visualized, so as to reconstruct the historical map of Ningbo's military defense system. For the convenience of calculation, the spatial data of each unit is inputted into the DEM map through GIS platform, based on the administrative maps of Ningbo in the Ming Dynasty depicted in The Historical Atlas of China [19].

Table 3 Hierarchy evaluation system of the coastal defense system's accessibility in the Ming Dynasty

\begin{tabular}{lcl}
\hline Overall objective & First-level indicators & Second-level indicators \\
\hline $\begin{array}{l}\text { Accessibility of the coastal } \\
\text { defense system }\end{array}$ & $\begin{array}{c}\text { Accessibility between beacon towers } \\
\text { and Wei-Suo (B1) }\end{array}$ & $\begin{array}{l}\text { Number of the beacon towers (C1) } \\
\text { Average observation distance of the } \\
\text { beacon towers (C2) }\end{array}$ \\
& $\begin{array}{ll}\text { Accessibility between post stations and field of the beacon towers (C3) } \\
\text { Wei-Suo (B2) }\end{array}$ & $\begin{array}{l}\text { Distance between post stations and } \\
\text { Wei-Suo (C4) }\end{array}$ \\
& Accessibility among Wei-Suo (B3) & $\begin{array}{l}\text { Distance of urgent delivery routes (C5) } \\
\text { Direct distance (C6) }\end{array}$ \\
\hline
\end{tabular}




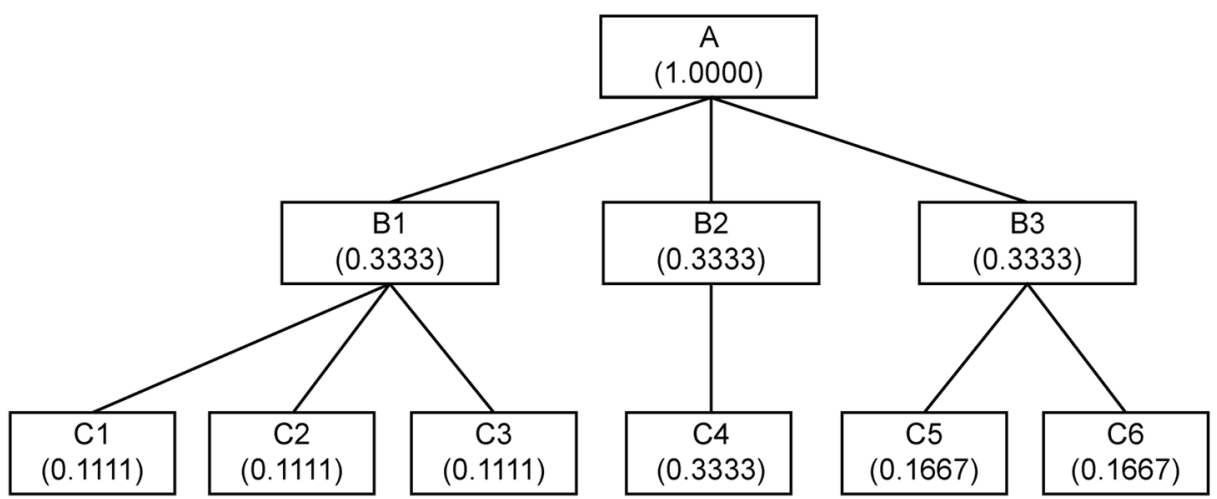

Fig. 2 Hierarchy evaluation model of the coastal defense system's accessibility in the Ming Dynasty

There are several ways to obtain geographic locations:

(1) For the detectable Wei-Suo and beacon towers, Global Positioning System (GPS) can be employed for investigation, and the national cultural relics census data also provides references of the position.

(2) Some beacon towers and post stations which have vanished can be localized by using the locations identified by previous researchers [16, 20, 26, 27, 29].

(3) For urgent delivery stations that are non-existent and untraceable, their approximate geographical scopes can be generalized first based on historical materials. Then, their current locations can be identified by searching their names in the modern administrative map, because many ancient names are still used as place names, such as "Chuanshan Pu" (穿山铺) in Ming Dynasty and “Chuanshan Village” (穿山村) today. If the place name can not be matched, it can be inferred from the locations of known units and the transmission routes. After that, all data are proofread to get the probable locations.

Although the visual reconstruction of the distribution map can not restore its then spatial form, it reproduces the basic location and spatial relationships of Ningbo's military defense system in the Ming Dynasty, contributing to an organic connection between fragmented historical data, and laying a foundation for overall intuitive spatial analysis, which is beyond the reach of pure character descriptions. The historical map can provide further analysis materials for this study (Fig. 3).

\subsubsection{Spatial data quantification}

Based on the reconstructed historical map, the spatial data of the military defense system in Ningbo of the Ming Dynasty can be quantified, which can be used as the attribute values of each indicator in the hierarchy evaluation model. Major GIS analysis tools used are as follows: Average Nearest Neighbor analysis for the calculation of the average distance between the two units; ViewShed analysis for calculating the visible area of beacon towers; Cost Path analysis for restoring the routes of post stations, urgent delivery stations and Wei-Suo.

(1) Average Nearest Neighbor analysis 


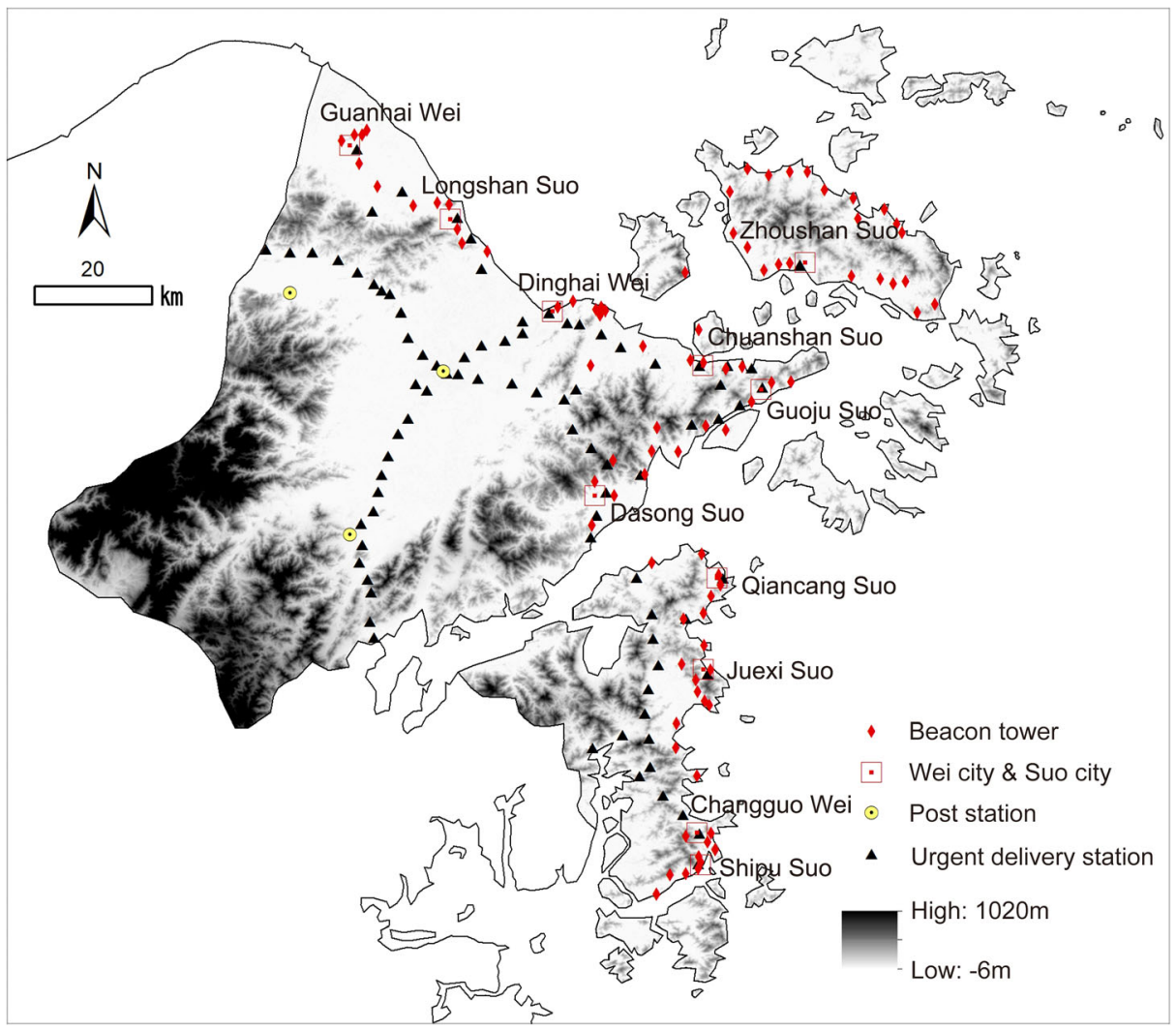

Fig. 3 Spatial distribution map of Ningbo's military defense system in the Ming Dynasty

Average Nearest Neighbor is an analysis method that can accurately and objectively determine the distribution pattern of a certain site. It was first proposed by the ecologist Clark and Evans in 1954 to study the distribution pattern of plants [3], and then introduced into the spatial distribution analysis of settlements. Several parameters are mentioned in the analysis results of the Average Nearest Neighbor, including Distribution Pattern, Observed Mean Distance, Nearest Neighbor Ratio, etc. In this study, the calculation results of Average Observation Distance are mainly used to obtain the average observation distance among beacon towers within the jurisdiction of a Wei city or Suo city.

The analysis process is as follows: taking the coordinates of beacon towers as the basic data, and making use of Average Nearest Neighbor in GIS tool to get the results. Table 4 presents the average observation distance statistics of Ningbo's beacon towers in the Ming Dynasty.

(2) Viewshed analysis

Viewshed aims to explore the general visual area of a special point in the space. The influencing factors include the terrain features of the observation points and around the visual range. In this study, the visual analysis method is used to analyze the visual situation and extract the visual field data of beacon towers. The analysis process is as follows:

(1) Taking the DEM data of Ningbo as the surface data, and the coordinates of beacon towers as the observation points, the visual area across the whole range is analyzed by 
Table 4 Average observation distance statistics of the beacon towers

\begin{tabular}{ll}
\hline Wei cities \& Suo cities & Average observation distance of beacon towers (Unit: km) \\
\hline Dasong Suo & 3.93 \\
Guanhai Wei & 2.30 \\
Longshan Suo & 2.28 \\
Dinghai Wei & 4.96 \\
Chuanshan Suo & 3.60 \\
Guoju Suo & 3.19 \\
Zhoushan Suo & 2.97 \\
Changguo Wei & 3.47 \\
Qiancang Suo & 3.23 \\
Juexi Suo & 2.33 \\
Shipu Suo & 1.88 \\
\hline
\end{tabular}

using "Viewshed" command. This study analyzes the visual field of beacon towers within the jurisdiction of each Wei city and Suo city, and the results are shown in Fig. 4. For each group of observation points, green zone refers to the visible area, and gray zone represents the invisible area.

(2) The raster-layer map is converted into polygon-layer map by using the "Raster to Polygon" command, and then the area of visual field can be calculated. The results are shown in Table 5.

\section{(3) Cost Path analysis}

The path between the two points is usually not a straight line. Since ancient times, the primary concern for people to choose a path is to minimize the traffic cost. Traffic cost is determined by humanistic and social factors, as well as geographical factors related to the slope, undulation and roughness of the terrain, which affect the physical strength and time required for human crossing, and then affect their road selection [2]. Due to the incompleteness of humanistic and social data, relevant parameters can not be accurately collected. Besides, the path studied just serves a single purpose, so only the terrain parameters are involved in the cost path analysis, namely, DEM data. In this study, Cost Path analysis is used to restore the paths among post stations, urgent delivery stations, Wei cities and Suo cities. The analysis process is as follows:

(1) DEM data are calculated by using "Slope" command to generate terrain slope data, and then slope data are reclassified into 10 categories with the help of "Reclassify" command to get "reclass-slope" data.

(2) DEM data are calculated by adopting "Focal statistics" command to generate rough terrain data, and then reclassified as above to get "reclass-rough" data.

(3) The "Raster Calculate" command is utilized to get the "cost-raster" data, the calculation formula is as follows:

"cost-raster" = "reclass-slope" * 0.6 + "reclass-rough" * 0.4 .

(4) Based on the "cost-raster" data, the spatial data of the starting point is calculated by using "Cost Distance" command, and the "cost-distance" data and "cost-backlink" data are obtained. 


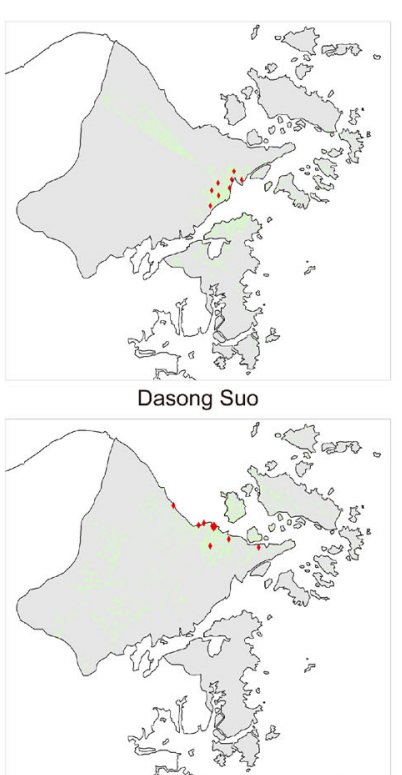

Dinghai Wei

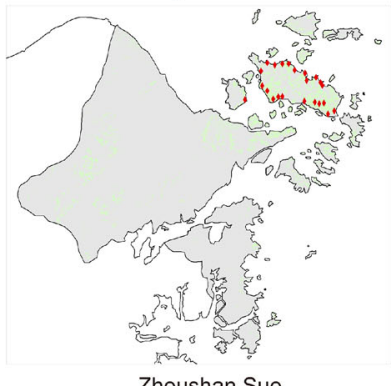

Zhoushan Suo

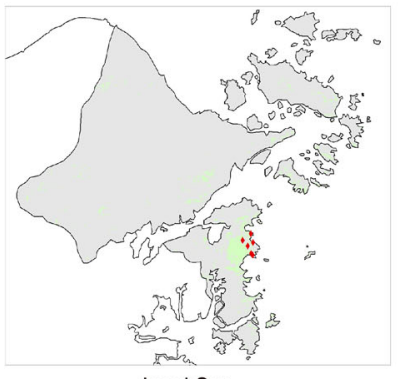

Juexi Suo
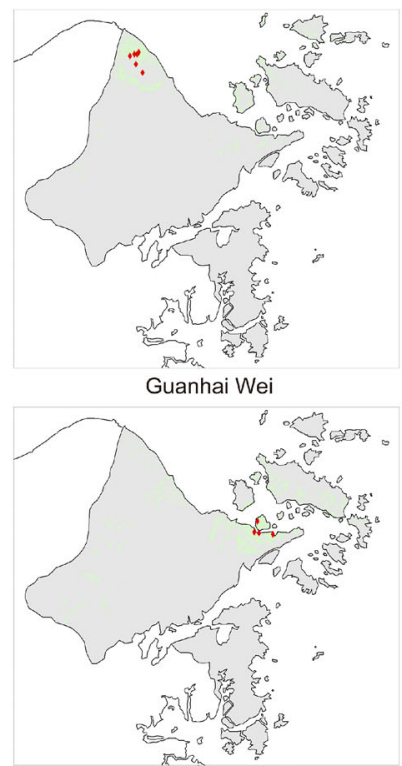

Chuanshan Suo

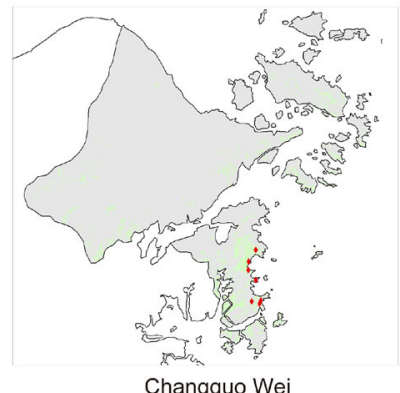

Changguo Wei

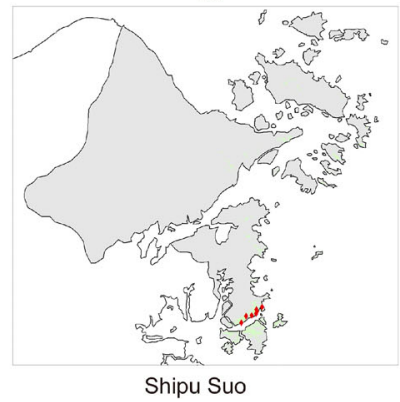

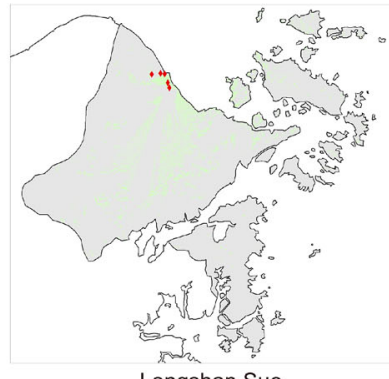

Longshan Suo

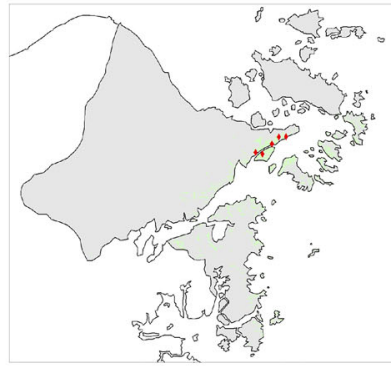

Guoju Suo

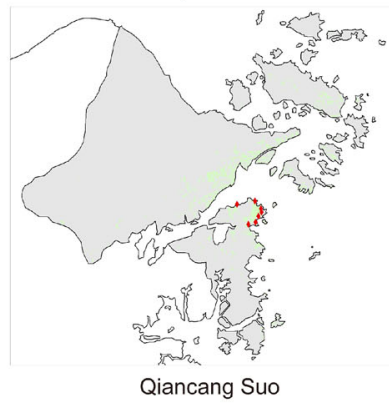

- Beacon tower

Invisible

Visible

Fig. 4 Viewshed analysis results of the beacon towers

(5) Finally, taking the terminal point as the calculation object, and loading the "costdistance" data and "cost-backlink" data by using the "Cost Path" command for calculations, the cost path from the starting point to terminal point is obtained. 
Table 5 Visual field statistics of the beacon towers

\begin{tabular}{ll}
\hline Wei cities \& Suo cities & Area of beacon towers' visual field $\left(\mathrm{Unit}^{\mathrm{k}} \mathrm{km}^{2}\right)$ \\
\hline Dasong Suo & 329.86 \\
Guanhai Wei & 203.24 \\
Longshan Suo & 482.37 \\
Dinghai Wei & 448.24 \\
Chuanshan Suo & 253.46 \\
Guoju Suo & 242.81 \\
Zhoushan Suo & 548.67 \\
Changguo Wei & 420.09 \\
Qiancang Suo & 259.51 \\
Juexi Suo & 259.10 \\
Shipu Suo & 105.59 \\
\hline
\end{tabular}

In this study, the paths among post stations, urgent delivery stations, Wei cities and Suo cities are restored respectively (Fig. 5), and the distance between the two nearest settlements is calculated as shown in Tables 6 and 7.

\subsection{Score unification}

Due to the inconsistent units of attribute values of these evaluation indicators, in order to unify the fractional magnitude, the attribute value of each evaluation indicator needs to be converted into the hundred-mark score.

To be specific, the attribute values of $\mathrm{C} 1$ and $\mathrm{C} 3$ are proportional to their scores. The following equation is adopted:

$$
F=\frac{c_{n}\left(f_{i}\right)}{c_{n}\left(f_{\max }\right)} \times 100
$$

The attribute values of $\mathrm{C} 2, \mathrm{C} 4, \mathrm{C} 5$ and $\mathrm{C} 6$ are inversely proportional to their scores. The equation is expressed as follows:

$$
F=\frac{c_{n}\left(f_{\text {min }}\right)}{c_{n}\left(f_{i}\right)} \times 100
$$

where $\mathrm{F}$ is the quantified score after quantization, $\mathrm{Cn}(\mathrm{fi})$ is the attribute value of the $\mathrm{i}$-th object in the evaluation indicator $\mathrm{Cn} ; \mathrm{Cn}$ (fmax) and $\mathrm{Cn}(\mathrm{fmin})$ are the maximum and maximum attribute values of the evaluation indicator an, respectively.

After the quantization, the sore $\mathrm{F}$ falls into the range of 0 to 100.

\section{Results and discussion}

The spatial data extraction results of the above evaluation indicators are substituted into Eqs. (1) or (2) for score quantification respectively, and the results are shown in Table 8.

Substituting the scores in Table 8 into the hierarchy evaluation model (Fig. 2), the final quantization results of accessibility of the military settlements can be obtained, as shown in Table 9. 


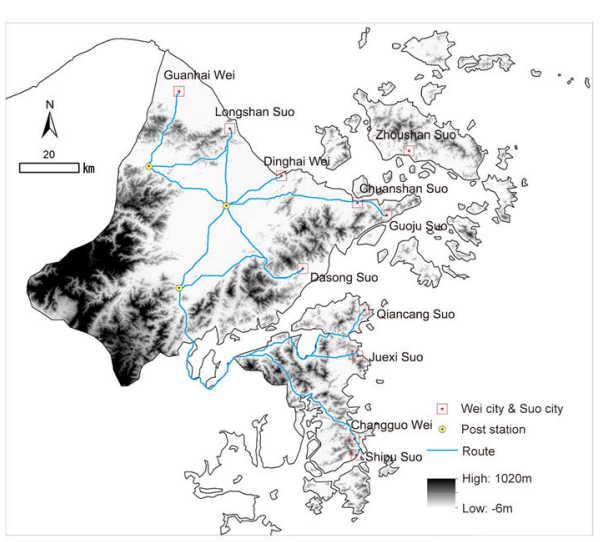

(a)

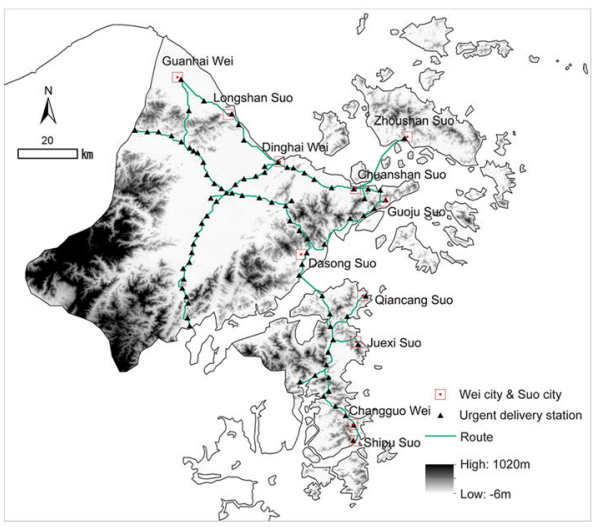

(b)

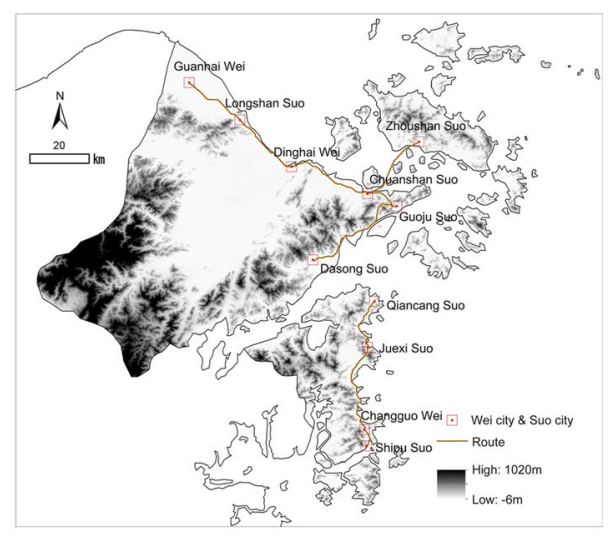

(c)

Fig. 5 Restoration of the paths among post stations (a), urgent delivery stations (b), Wei cities and Suo cities (c)

By using the tool "Inverse Distance Weight" on GIS to project the scores of the above indicators into the historical map, the visualization results of the military settlements' accessibility of Ningbo in Ming Dynasty can be obtained (Fig. 6). It can be seen that the accessibility of military settlements in different areas of Ningbo is generally characterized by

Table 6 Distance statistics among the post stations, Wei cities and Suo cities

\begin{tabular}{lll}
\hline Wei cities \& Suo cities & The nearest post stations & Distance (Unit: km) \\
\hline Dasong Suo & Siming Yi & 37.50 \\
Guanhai Wei & Chejiu Yi & 25.55 \\
Longshan Suo & Siming Yi & 23.87 \\
Dinghai Wei & Siming Yi & 18.71 \\
Chuanshan Suo & Siming Yi & 40.23 \\
Guoju Suo & Siming Yi & 51.36 \\
Zhoushan Suo & $/$ & $/$ \\
Changguo Wei & Lianshan Yi & 102.34 \\
Qiancang Suo & Lianshan Yi & 105.88 \\
Juexi Suo & Lianshan Yi & 95.37 \\
Shipu Suo & Lianshan Yi & 108.69 \\
\hline
\end{tabular}


Table 7 Distance statistics among Wei cities and Suo cities

\begin{tabular}{llll}
\hline $\begin{array}{l}\text { Wei cities \& Suo } \\
\text { cities }\end{array}$ & $\begin{array}{l}\text { The nearest Wei cities or Suo } \\
\text { cities }\end{array}$ & $\begin{array}{l}\text { Distance of urgent delivery paths } \\
(\text { Unit: } \mathrm{km})\end{array}$ & $\begin{array}{l}\text { Direct distance (Unit: } \\
\mathrm{km})\end{array}$ \\
\hline Dasong Suo & Guoju Suo & 37.81 & 35.74 \\
Guanhai Wei & Longshan Suo & 19.18 & 19.43 \\
Longshan Suo & Guanhai Wei & 19.18 & 19.43 \\
Dinghai Wei & Longshan Suo & 21.93 & 21.38 \\
Chuanshan Suo & Guoju Suo & 13.76 & 10.78 \\
Guoju Suo & Chuanshan Suo & 13.76 & 10.78 \\
Zhoushan Suo & Chuanshan Suo & 22.29 & 22.60 \\
Changguo Wei & Shipu Suo & 6.25 & 5.93 \\
Qiancang Suo & Juexi Suo & 28.63 & 17.64 \\
Juexi Suo & Qiancang Suo & 28.63 & 17.64 \\
Shipu Suo & Changguo Wei & 6.25 & 5.93 \\
\hline
\end{tabular}

the following: from the regional perspective, it shows high accessibility in northern and southern areas but low accessibility in central areas; from the settlement level perspective, the accessibility of Wei cities is generally higher than that of Suo cities (Fig. 6a). Separately, the accessibility between beacon towers in Wei-Suo is found the highest in Zhoushan Suo, followed by Longshan Suo, and the rest is lower (Fig. 6b); the accessibility between post stations and Wei-Suo is higher in inland and lower in coastal area (Fig. 6c); Changguo Wei and Shipu Suo are identified with the highest accessibility in terms of the accessibility among Wei-Suo, while the others are at a lower level (Fig. 6d).

That the underlying reasons are shown below: first, from the military point of view: for the sake of unified management, the coastal military defense system in the Ming Dynasty was designed with strict military hierarchies. Wei cities, the military settlements of the highest level, managed a large range of defense issues around, with tens of thousands of soldiers stationed. Suo cities were under the jurisdiction of Wei cities. Although they could also undertake the task of defense independently, Suo cities needed to follow the dispatch of Wei cities and cooperated with each other in case of major combats. The higher the accessibility of the Wei city, the easier it was to transmit information to the Suo cities and commanded operations. Therefore, the accessibility of the Wei city was generally higher than that of Suo city, which was in agreement with the military management of the Ming Dynasty. From the perspective of geographical features: being surrounded by the sea and hundreds of islands,

Table 8 Quantization results statistics of the evaluation indicators

\begin{tabular}{lllllll}
\hline Wei cities \& Suo cities & C1 & C2 & C3 & C4 & C5 & C6 \\
\hline Dasong Suo & 34.78 & 47.84 & 60.12 & 49.89 & 16.53 & 16.59 \\
Guanhai Wei & 26.09 & 81.74 & 37.04 & 73.23 & 32.59 & 30.52 \\
Longshan Suo & 21.74 & 82.46 & 87.92 & 78.38 & 32.59 & 30.52 \\
Dinghai Wei & 52.17 & 37.90 & 81.70 & 100 & 28.50 & 27.74 \\
Chuanshan Suo & 17.39 & 52.22 & 46.20 & 46.51 & 45.42 & 55.01 \\
Guoju Suo & 21.74 & 58.93 & 44.25 & 36.43 & 45.42 & 55.01 \\
Zhoushan Suo & 100 & 63.30 & 100 & 0 & 28.04 & 26.24 \\
Changguo Wei & 39.13 & 54.18 & 76.57 & 18.28 & 100 & 100 \\
Qiancang Suo & 30.43 & 58.20 & 47.30 & 17.67 & 21.83 & 33.62 \\
Juexi Suo & 30.43 & 80.69 & 47.22 & 19.62 & 21.83 & 33.62 \\
Shipu Suo & 21.74 & 100 & 19.24 & 17.21 & 100 & 100 \\
\hline
\end{tabular}


Table 9 Final quantization results statistics

\begin{tabular}{lllll}
\hline Wei cities \& Suo cities & B1 & B2 & B3 & Quantization results \\
\hline Dasong Suo & 47.58 & 49.89 & 16.56 & 38.01 \\
Guanhai Wei & 48.29 & 73.23 & 31.56 & 51.02 \\
Longshan Suo & 64.04 & 78.38 & 31.56 & 57.99 \\
Dinghai Wei & 57.26 & 100 & 28.12 & 61.79 \\
Chuanshan Suo & 38.60 & 46.51 & 50.22 & 45.11 \\
Guoju Suo & 41.64 & 36.43 & 50.22 & 42.76 \\
Zhoushan Suo & 87.77 & 0 & 27.14 & 38.30 \\
Changguo Wei & 56.63 & 18.28 & 100 & 58.31 \\
Qiancang Suo & 45.31 & 17.67 & 27.73 & 30.23 \\
Juexi Suo & 52.78 & 19.62 & 27.73 & 33.37 \\
Shipu Suo & 46.99 & 17.21 & 100 & 54.74 \\
\hline
\end{tabular}

Zhoushan Suo area was a convenient hiding place for Japanese pirates; due to a wide field of vision, it was conducive to build beacon towers to observe the enemy's situation nearby, so

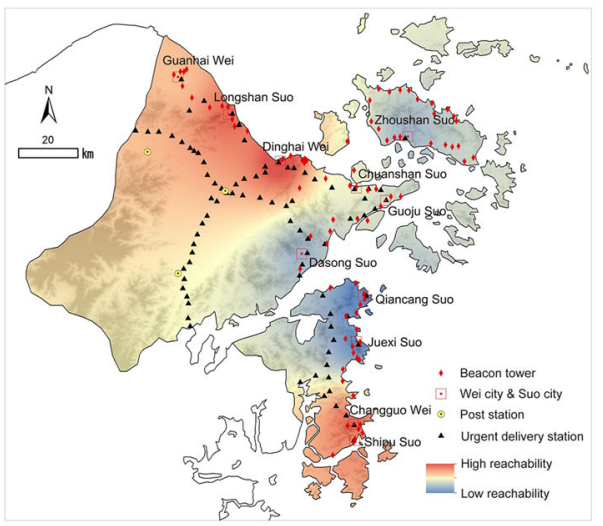

(a)

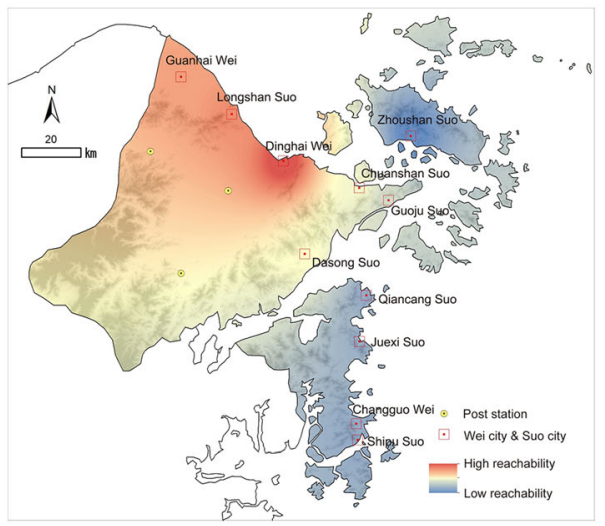

(c)

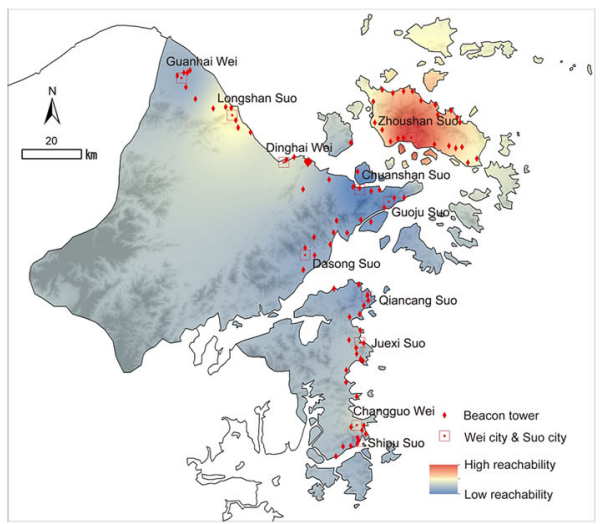

(b)

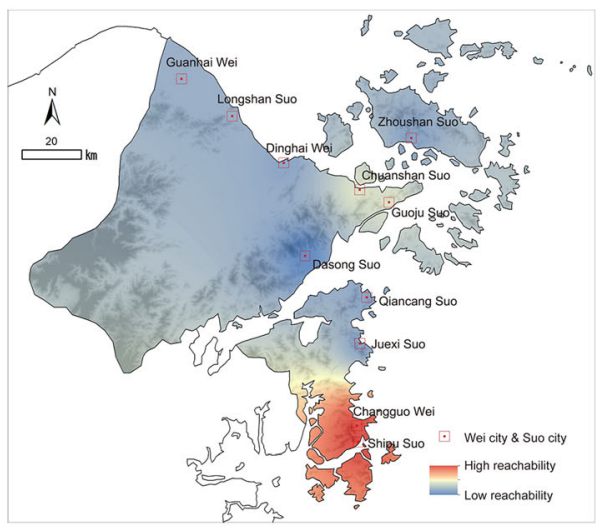

(d)

Fig. 6 Visualization results of the accessibility: overall accessibility of the military defense system (a), accessibility between beacon towers and Wei-Suo (b), accessibility between post stations and Wei-Suo (c) and accessibility among Wei-Suo (d) 
that Zhoushan Suo boosted the highest accessibility between beacon towers and Wei-Suo. The northern inland terrain of Ningbo was relatively flat, which was suitable for the construction of post facilities. While the coastal areas were blocked by mountains with rugged roads, which restricted long-distance transmission, so the accessibility between post stations and Wei-Suo in the inland area was higher than that of coastal areas. In contrast, the accessibility among WeiSuo had much to do with their distribution density.

Combined with the geographical features and historical situation of Ningbo, it can be seen that the characteristics of the accessibility of Ningbo's military settlements in Ming Dynasty were basically in agreement with history, suggesting that the accessibility was not only affected by geographical conditions, but also took into account military and political needs. Yet people can not be informed of such information from the original historical data. From Tables 1 and 2 to Fig. 6, the historical data is finally showed in a visual way after the processing of reconstruction, spatial analysis and calculation, hierarchical quantization and data projection, which makes us know the military settlements' accessibility and reveals the distribution characteristics and reasons of Ningbo's military defense system in Ming Dynasty. At the same time, the results show that the combined research method of quantification and visualization used in this study is scientific for the analysis of the attribute characteristics.

Visualization means "reprocessing" of historical data, which is a new exploration process of historical data collected [22]. The combined research method of quantification and visualization with the computer-aid can be used for data extraction and in-depth analysis to present people with history hidden in local chronicles, historical records and maps in a more clearly and intuitively manner. When the research also relates to the target field of other scholars, visualization will greatly reduce the difficulty and time of understanding, so as to enhance inter-disciplinary communication.

\section{Conclusions}

Based on the visual reconstruction of historical data, the accessibility diagram of Ningbo's military defense system in the Ming Dynasty is investigated, whose geographic information is characterized by concrete and intuitive performance, multidimensional information interconnections, and wide and rich information content, further exploring the hidden value of historical data. The unique significance of the proposed method is highlighted in the research of complex systems, serving as an effective way to interdisciplinary study of natural and historical geography, and comprehensive study of historical environment. Although there is a certain gap in accuracy compared to real-time remote sensing images, the visualization diagram of text-based geographic information is drawn by strictly following objective historical records. Therefore, this method is of good historical authenticity and scientific rationality; moreover, it also provides a possibility for spatial correlation analysis which cannot be carried out by descriptive research. It is undoubtedly of great significance to enriching the research methods of historical geography and expanding the research field of historical spatial analysis.

By using the combined method of quantification and visualization, this study not only evaluates the overall accessibility of the coastal military defense system of Ningbo, but also identifies the accessibility among beacon towers, post stations, Wei cities and Suo cities, so that the internal attribute characteristics of the coastal military defense system can be presented in a graphical way, which is very clear and intuitive. Method demonstration reveals that it brings out the results that cannot be obtained by intuitive feeling, and is characterized by clear methods, easy operation, and 
high repeatability. Therefore, the method is highly suitable for evaluating Ningbo's military defense system in Ming Dynasty, and can also be widely used in the study of complex system in historical period, so as to facilitate people's understanding of history.

Acknowledgments Thanks to the National Natural Science Foundation of China (grant no. 52078324 and 51778400) and Major Research on Philosophy and Social Sciences of the Ministry of Education of China (grant no. 18JZD059 and 19JZD056) for their funding of this study. Thanks to the China Scholarship Council for their support of this study.

\section{Declarations Not applicable.}

Open Access This article is licensed under a Creative Commons Attribution 4.0 International License, which permits use, sharing, adaptation, distribution and reproduction in any medium or format, as long as you give appropriate credit to the original author(s) and the source, provide a link to the Creative Commons licence, and indicate if changes were made. The images or other third party material in this article are included in the article's Creative Commons licence, unless indicated otherwise in a credit line to the material. If material is not included in the article's Creative Commons licence and your intended use is not permitted by statutory regulation or exceeds the permitted use, you will need to obtain permission directly from the copyright holder. To view a copy of this licence, visit http://creativecommons.org/licenses/by/4.0/.

\section{References}

1. Bentley E (2012) A historical atlas in narrative form. Cartographic and Geographic Information Science 39(4):219-231. https://doi.org/10.1559/15230406394219

2. Cao Y, Zhang Y, Zhang H (2014) GIS-based road rehabilitation of the map on the Datong zone of the Great Wall defense system in Ming dynasty. Journal of Agricultural University of Hebei 02:138-144

3. Clark PJ, Evans FC (1954) Distance to nearest neighbor as a measure of spatial relationships in populations. Ecology 35(4):445-453. https://doi.org/10.2307/1931034

4. Fan Z (1990) Research on coastal defense in Ming dynasty. Hist Res 03:44-54

5. Gong S (1993) A preliminary study on variations of the distribution of Zhang-disease. Acta Geograph Sin 04:304-316

6. Guan WW, Bol PK et al (2012) WorldMap - a geospatial framework for collaborative research. Ann GIS 18(2):121-134. https://doi.org/10.1080/19475683.2012.668559

7. Hansen WG (1959) How accessibility shapes land use. Journal of American Institute of Planners 25:73-76. https://doi.org/10.1080/01944365908978307

8. Institute of Geographical Sciences (1987) Population atlas of China. China Statistics Press, Beijing

9. Lin C (1981) On the nature of geography. Sci Geogr Sin 02:97-104

10. Liu J, Zhang Y, Cao Y (2013) Study on the defense system of Ming's Great Wall based on the reachable domain. Journal of Architecture S1:108-111

11. Oldenderfer M, Maschner HDG (1996) Anthropology, space, and geographic information systems. Oxford University Press, Oxford

12. Pawson E (1997) The New Zealand historical atlas. J Hist Geogr 23:496-499

13. Pearce MW (2008) Framing the days: place and narrative in cartography. Cartogr Geogr Inf Sci 35(1):1732. https://doi.org/10.1559/152304008783475661

14. Piatti B, Bär HR, Reuschel AK, Hurni L, Cartwright W (2009) Mapping literature: towards a geography of fiction. In: Cartography and Art. Lecture Notes in Geoinformation and Cartography. Springer, Berlin, Heidelberg. https://doi.org/10.1007/978-3-540-68569-2_15

15. Shi H (1996) The historial atlas of Xi'an. Xi'an Maps Press, Xi'an

16. Shi Y (2018) Wind and rain are far away, beacons of vicissitudes are near. Zhoushan Daily 04-03:005

17. Staley DJ (2002) Computers, visualization, and history: how new technology will transform our understanding of the past. M.E. Sharpe, New York

18. St-Hilaire M, Moldofsky B, Richard L, Beaudry M (2007) Geocoding and mapping historical census data: the geographical component of the Canadian century research infrastructure. Historical Methods 40:76-91. https://doi.org/10.3200/HMTS.40.2.76-91

19. Tan Q (1982) The historical atlas of China. Sino Maps Press, Beijing 
20. Tan L, Zhang Y, Lin Z (2018) Study on the spatial distribution of the post - delivery system in the Ming Dynasty. City Planning Review 42:92-96+140

21. Wang T (2010) Research on the relationship between the Great Wall of Datong Zhen in the Ming dynasty and natural geographical environment. Southeast University, Dissertation

22. Wang $Z$ (2017) Historical spatial data visualization and economic history study. Researches In Chinese Economic History 05:28-44+57

23. Wang J, Li M, Liu Y et al (2015) Visual expression of temporal and spatial evolution of historical events in the Nansha Islands. Sci Geogr Sin 35:608-614

24. Wu Y (1995) The historical atlas of counter-Japanese war. Sino Maps Press, Beijing

25. Yang Z (1994) Research on the post station in Ming dynasty. Shanghai Ancient Books Press, Shanghai

26. Yang Z (2020) Study on the defense efficiency of the coastal defense military settlements in the Ming dynasty. Tianjin University, Tianjin

27. Yin Z (2016) Studies on the coastal defense forts system of the Ming dynasty. Tianjin University, Dissertation

28. Zhang S (1560) Ningbo annals of Jiajing period.

29. Zhang Y (2012) Research on coastal defense of Ming and Qing dynasties in Ningbo. Ningbo Publishing House, Ningbo

30. Zheng R (2007) Chou Hai Tu Bian. Zhonghua Book Company, Beijing

Publisher's note Springer Nature remains neutral with regard to jurisdictional claims in published maps and institutional affiliations. 